\title{
MicroRNA-135b promotes proliferation, invasion and migration of osteosarcoma cells by degrading myocardin
}

\author{
WEI-GUO XU ${ }^{1 *}$, YING-LIE SHANG ${ }^{2,3^{*}}$, XIAN-RUI CONG ${ }^{1}$, XIA BIAN $^{4}$ and ZHEN YUAN ${ }^{3}$ \\ ${ }^{1}$ Department of Orthopedics, Shanxian County Dongda Hospital, Shanxian, Heze, Shandong 274300; \\ ${ }^{2}$ Department of Orthopedics, The 2nd People's Hospital of Dongying, Dawang Town, Dongying, Shandong 257335; \\ ${ }^{3}$ Department of Orthopedics, Qianfoshan Hospital, Shandong University, Jinan, Shandong 250014; \\ ${ }^{4}$ Department of Oncology, The People's Hospital of Dongying, Dongying District, \\ Dongying, Shandong 257091, P.R. China
}

Received April 30, 2014; Accepted June 23, 2014

DOI: $10.3892 /$ ijo.2014.2641

\begin{abstract}
MicroRNA-135b (miR-135b) functions as an oncogene in malignant tumors suggesting that it might be a target for malignant tumor therapy, but its role in osteosarcoma has not been investigated to date. In this study, we found that myocardin expression is specifically attenuated in MG63 osteosarcoma cells. Silencing miR-135b restores the expression of myocardin and miR-135b inhibits the expression of myocardin by targeting its 3'UTR in MG63 osteosarcoma cells. In additional experiments, we found that miR-135b was highly expressed in osteosarcoma tissues and it promoted MG63 cell proliferation, migration and invasion. Myocardin had the opposite effects of miR-135b, which suppressed proliferation, migration and invasion in MG63 cells. Thus, it is probably that miR-135b promotes proliferation, invasion and migration of osteosarcoma cells by degrading myocardin.
\end{abstract}

\section{Introduction}

Although osteosarcomas have been treated with chemotherapy for $>30$ years, patients with recurrent or metastatic osteosarcomas still have very poor prognosis (1-3). Finding new strategies to treat recurrent or metastatic osteosarcoma remains an important but unmet clinical need.

Myocardin has been reported to be a key regulator of cardiac and smooth muscle differentiation and its expression is restricted to smooth and cardiac muscle lineages (4). Myocardin drives transcription through the interaction with the MADS box-containing transcription factor, serum response factor (SRF), which binds to a consensus DNA

Correspondence to: Dr Zhen Yuan, Department of Orthopedics, Qianfoshan Hospital, Shandong University, 16766 Jingshi Road, Jinan, Shandong 250014, P.R. China

E-mail: devinyuanzhen@163.com; jinding2014@gmail.com

*Contributed equally

Key words: miR-135b, myocardin, osteosarcoma sequence, $\mathrm{CC}[\mathrm{A} / \mathrm{T}]_{6} \mathrm{GG}$ (CArG box) $(5,6)$. In addition, myocardin functions as an effective inducer of growth arrest and differentiation of some tumor and is frequently repressed during human malignant transformation (7). Although the importance of myocardin in cardiac and smooth muscle has firmly established, its role in osteosarcomas is poorly understood, let alone why it cannot be expressed in osteosarcoma.

Recently, deregulation of expression of several miRNAs has been identified between osteosarcoma samples and normal human osteoblasts as well as several important studies have focused on the impact of microRNAs (miRNA, miR) on tumorigenesis and progression of osteosarcoma (8-13). The expression of miR-135b is upregulated in osteosarcoma (8) and miR-135b functions as an oncogene in other malignant tumors suggesting that it might be a target for malignant tumor therapy (14-18), but its roles still keep emerging in osteosarcoma.

Our results demonstrated that myocardin expression is specifically attenuated in osteosarcoma MG63 cells. We next performed an analysis of potential microRNA target sites employing 3 commonly used prediction algorithms, miRanda, TargetScan and miRDB. All 3 algorithms predicted that miR-135a and miR-135b can target 3'UTR of myocardin. However, results of microarray assay and real-time PCR showed that only miR-135b was significantly upregulated in osteosarcoma tissues. Thus, we reasoned that due to the overexpression of miR-135b, myocardin was suppressed or degraded by miR-135b in osteosarcoma. Following experiments showed that miR-135b degraded myocardin mRNA through targeting its 3'UTR in osteosarcoma MG63 cells. Moreover, miR-135b promoted proliferation, invasion and migration of MG63 cells. Finally, we found that myocardin has the opposite effects of miR-135b, which suppressed proliferation, migration and invasion in MG63 cells. Thus, it is probably that miR-135b promotes proliferation, invasion and migration of osteosarcoma cells by degrading myocardin.

\section{Materials and methods}

Cell culture and expression plasmids. MG63 human osteosarcoma cells were maintained in minimum essential medium (Life Technologies, Inc., Carlsbad, CA, USA) containing 
$10 \%$ fetal bovine serum, $1 \%$ non-essential amino acids, and $1 \%$ penicillin/streptomycin. A7r5 cells were maintained as described previously (19). All expression plasmids were purchased from Tiangene (Tianjin, China). Briefly, the pcDNA3.1-myocardin contains 3'UTR of myocardin (1214nt) and a cDNA encoding amino acids 1-935 of mouse myocardin, named myocardin-WT. Site-directed mutagenesis of the miR-135b target-site in the myocardin-3'UTR was carried out using Quik change-mutagenesis kit (Stratagene, Heidelberg, Germany), myocardin-WT as a template. For transfection experiments, the cells were cultured in serum-free medium without antibiotics at $60 \%$ confluence for $24 \mathrm{~h}$, and then transfected with transfection reagent (Lipofectamine 2000, Invitrogen, Carlsbad, CA, USA) according to the manufacturer's instructions. After incubation for $6 \mathrm{~h}$, the medium was removed and replaced with normal culture medium for $48 \mathrm{~h}$.

miRNA precursors, anti-miRNA oligonucleotides. The locked nucleic acid (LNA)-modified oligonucleotide inhibitors (antimiR-135b) used for miRNA knock-down and scramble were purchased from Exiqon. The miR-135b miRNA precursor (pre-miR-135b) and a control precursor (scramble) were purchased from Ambion, Inc.

Reverse transcription-polymerase chain reaction. Total RNA was isolated from cells using TRIzol reagent (Invitrogen). cDNA was synthesized from $1 \mu \mathrm{g}$ of total RNA in a $20-\mu \mathrm{l}$ reverse transcription (RT) system followed by PCR amplification in a 50- $\mu$ l PCR system performed using an RT-PCR kit (Promega, Madison, WI, USA). Housekeeping gene glyceraldehyde-3-phosphate dehydrogenase (GAPDH) was used as RNA loading control. The PCR primer sequences are as follows: myocardin: 5'-GGACTGCTCTGGCAACCCAGTGC'; 5'-CATCTGCTGACTCCGGGTCATTTGC-3'. The PCR was conducted according to the manufacturer's instructions and the PCR products were analyzed by agarose gel electrophoresis. Gels were photographed and densities of the bands were determined with a computerized image analysis system (Alpha Innotech, San Leandro, CA, USA). The area of each band was calculated as the integrated density value (IDV). Mean values were calculated from three separate experiments. The IDV ratio of myocardin to GAPDH was calculated for each sample.

Cell counting assay. Forty-eight hours after transfection, MG63 cells were seeded in 96-well plates in triplicate at a density of $5 \times 10^{3}$ cells/well in $100 \mu \mathrm{l}$ of RPMI-1640 medium containing $10 \% \mathrm{FBS}$ and antibiotics. Cell proliferation was evaluated using Cell Counting Kit-8 (Dojindo Lab, Kumamoto), according to the manufacturer's instructions and the absorbance value for each well was measured at $450 \mathrm{~nm}$ using a microplate reader (Spectra Max 180, Molecular Devices).

MTT assay. The effect on the cell proliferation was assessed by 3-(4, 5-dimethylthiazol-2-yl)-2, 5-diphenyltetrazolium (MTT; Sigma, St. Louis, MO, USA) assay was performed as described before (20). Absorbance was directly proportional to the number of survival cells.

BrdU proliferation analysis. Cell proliferation was assessed by using a colorimetric BrdU proliferation kit according to the manufacturer's instructions (Roche, Indianapolis, IN, USA). The cells transfected with myocardin or empty vector or pre-miR-135b or anti-miR-135b were labeled with BrdU for 3-4 h. The genomic DNA was fixed and denatured, and then incubated with peroxidase-conjugated anti-BrdU antibody for $90 \mathrm{~min}$. A substrate for the conjugated peroxidase was then added and the reaction product was quantified by measuring the absorbance. The results were then normalized by the number of total viable cells.

Migration and invasion assay. For Transwell migration assays, $2.5 \times 10^{4}-5.3 \times 10^{4}$ cells were plated in the top chamber with the non-coated membrane (24-well insert; pore size, $8 \mathrm{~mm}$; BD Biosciences). For invasion assays, $1.25 \times 10^{5}$ cells were plated in the top chamber with Matrigel-coated membrane (24-well insert; pore size, $8 \mathrm{~mm}$; BD Biosciences). In both assays, cells were plated in medium without serum or growth factors, and medium supplemented with serum was used as a chemoattractant in the lower chamber. The cells were incubated for $24 \mathrm{~h}$ and cells that did not migrate or invade through the pores were removed by a cotton swab. Cells on the lower surface of the membrane were stained with the Diff-Quick Staining Set (Dade) and counted.

Western blot analysis. Western blot analysis was performed as described before (21). Antibodies used are listed in Table I.

miRNA microarray. Total RNA from cultured cells, with efficient recovery of small RNAs, was isolated using the mirVana miRNA Isolation kit (Ambion, Austin, TX, USA). cRNA for each sample was synthesized by using 3' IVT Express kit (Affymetrix) according to the manufacturer's protocols. The purified cRNA was fragmented by incubation in fragmentation buffer (provided in the 3'IVT express kit) at $95^{\circ} \mathrm{C}$ for $35 \mathrm{~min}$ and chilled on ice. The fragmented labeled cRNA was applied to MicroRNA2.0 array (Affymetrix) and hybridized in Genechip hybridization oven 640 (Affymetrix) at $45^{\circ} \mathrm{C}$ for $18 \mathrm{~h}$. After washing and staining in Genechip fluidics station 450 (Affymetrix), the arrays were scanned by using Genechip scanner 3000 (Affymetrix). The gene expressions levels of samples were normalized and compared by using Partek GS 6.5 (Partek). Average-linkage hierarchical clustering of the data was applied by using the Cluster and the results were displayed by using TreeView.

Real-time PCR. Total RNA from cultured cells, with efficient recovery of small RNAs, was isolated using the mirVana miRNA Isolation kit (Ambion). Detection of the mature form of miRNAs was performed using the mirVana qRT-PCR miRNA Detection kit and qRT-PCR primer sets, according to the manufacturer's instructions (Ambion). The U6 small nuclear RNA was used as an internal control.

Gene microarray. Preparation of RNA from cells and analysis of Affymetrix gene chip microarray data were as previously described (22). Total RNA was prepared using TRIzol reagent (Invitrogen) according to the manufacturer's instructions. RNA was further purified using RNeasy columns (Qiagen, Chatsworth, CA, USA) and treatment with RNase-free DNase I (Qiagen). Total RNA was used to generate cRNA, which was 
Table I. Antibody used in the western blot assay.

\begin{tabular}{lr}
\hline Primary antibody & Secondary antibody \\
\hline Anti-CDK1 $(1: 500 ;$ Abcam $)$ & $\begin{array}{r}\text { Anti-rabbit secondary } \\
\text { antibodies (Li-COR) }\end{array}$ \\
Anti-CDK2 $(1: 500 ;$ Abcam) & \\
Anti-c-myc $(1: 200 ;$ Abcam $)$ & \\
Anti-PCNA (1:500; Abcam) & \\
Anti-Ki67 (1:500; Abcam) & \\
Anti-p53 (1:500; Abcam) & \\
Anti-p27 (1:500; Abcam) & \\
Anti-myocardin $(1: 200 ;$ Abcam $)$ & \\
Anti- $\beta$-actin $(1: 500 ;$ Abcam $)$ & \\
\hline
\end{tabular}

labeled with biotin as recommended by Affymetrix. cRNA was then hybridized to Affymetrix Hu95A GeneChips, which contain $\sim 12,000$ human oligonucleotide probe sets. After washing, the chips were scanned and analyzed using Microarray Suite 5.0 (Affymetrix). Average intensities for each Gene Chip were globally scaled to a target intensity of 150. Further analysis was performed using Gene Spring software version 5.0.1 to obtain expression level information, fold change, and P-values for each gene relative to control.

Luciferase reporter assay. The 3' untranslated region (3'UTR) of human myocardin mRNA was cloned in between the Not1 and Xba1 sites of pRL-TK (Promega) using PCR-generated fragment. Site-directed mutagenesis of the miR-135b targetsite in the myocardin-3'UTR was carried out using Quik change-mutagenesis kit (Stratagene), with myocardin-WT-luc as a template. For reporter assays, MG63 was transiently transfected with WT or mutant reporter plasmid and microRNA using Lipofectamine 2000 (Invitrogen). Reporter assays were performed $36 \mathrm{~h}$ post-transfection using the Dualluciferaseassay-system (Promega), normalized for transfection efficiency by cotransfected Renilla-luciferase.

Northern blot analysis. Northern blot analysis of miRNAs, were performed as described previously (23). Probes were labeled with $\left[\gamma^{32} \mathrm{P}\right]$-ATP complementary to miR-135b and U6 snRNA.

\section{Results}

Myocardin expression is specifically attenuated in osteosarcoma MG63 cells. To identify the level of myocardin expression, we performed RT-PCR and western blotting in A7r5 cells (as a positive control) as well as MG63 cells. We could not detect any myocardin mRNA (Fig. 1A) and protein expression (Fig. 1B) in MG63 cells, although stable expression of myocardin could be detected in A7r5 cells. Because myocardin functions as an effective inducer of growth arrest and differentiation of some tumor and is frequently repressed during human malignant transformation (7), we reasoned that the defect of myocardin was associated with pathogenesis and progression of osteosarcoma. To test this hypothesis and study biological effects of myocardin, we transfected MG63 cells
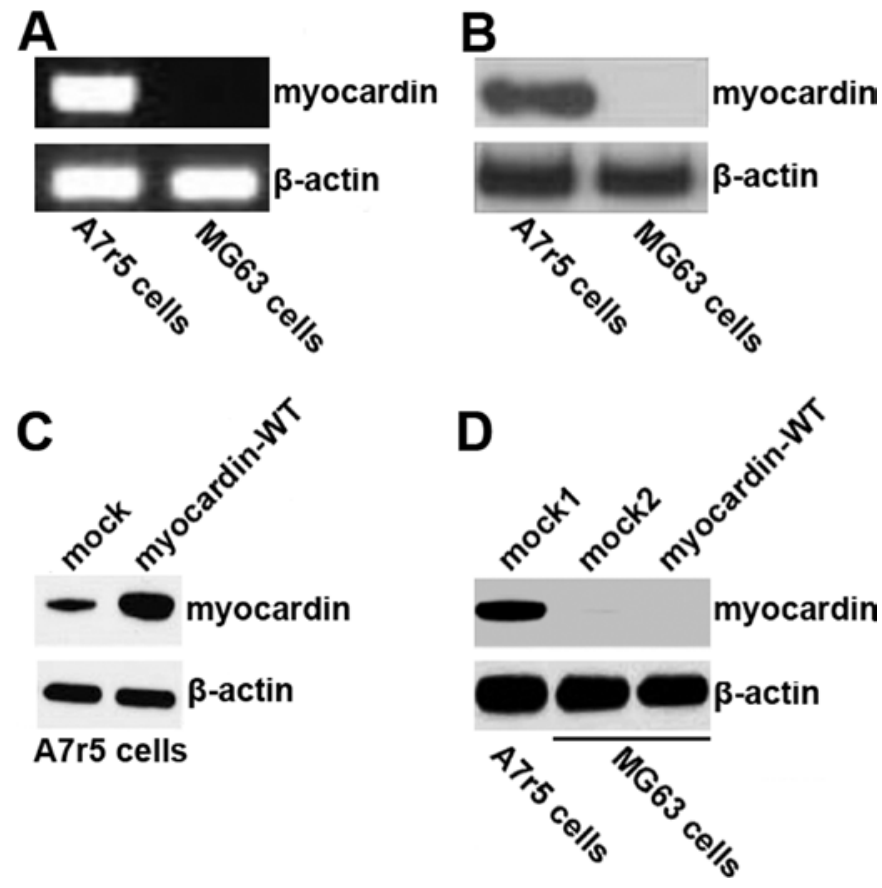

Figure 1. (A) RT-PCR for myocardin in A7r5 cells and MG63 cells. GAPDH was a loading control. $n=3$. (B) Western blotting for myocardin in A7r5 cells and MG63 cells. $\beta$-actin was a loading control. $n=3$. (C) Western blotting for myocardin in A7r5 cells. Mock groups were transfected with empty vector. $\beta$-actin was a loading control. $n=3$. (D) Western blotting for myocardin in A7r5 cells and MG63 cells. Mock1 and mock2 were transfected with empty vector. $\beta$-actin was a loading control. $n=3$.

with myocardin-WT expression plasmids. Although myocardin protein was significantly increased by myocardin-WT plasmids in A7r5 cells (Fig. 1C), we did not detect any protein expression of myocardin in MG63 cells (Fig. 1D).

Myocardin as a potential target of miR-135b in osteosarcoma MG63 cells. Having demonstrated that myocardin expression is specifically attenuated in MG63 cells and myocardin-WT plasmids cannot be expressed in the cells, we investigated the mechanisms inhibiting myocardin expression. MicroRNAs (miRNAs) are a new class of small ( 22 nucleotide) noncoding RNAs that negatively regulate protein-coding gene expression by targeting mRNA degradation or translation inhibition (24). Thus, we considered that myocardin was degraded by miRNA.

As a further confirmation, we used 3 common prediction algorithms - miRanda (http://www.microrna.org/), TargetScan (http://www.targetscan.org) and miRDB (http://mirdb.org/miRDB/index.html) to analyze 3'UTR of myocardin. All 3 algorithms predicted that miR-135a and miR-135b could target 3'UTR of myocardin (Fig. 2A) and the predicted target is shown in Fig. 2B. We also performed microRNA profiling and real-time PCR to detect and analyze the difference of miRNA profiling in A7r5 cells (myocardinpositive cells) and MG63 cells (myocardin-negative cells). The results of the microarray (Fig. 2C) showed that miR-135b expression was significantly upregulated in MG63 cells. To confirm the results of microarray, we performed real-time PCR to analyze the level of miR-135b expression between 

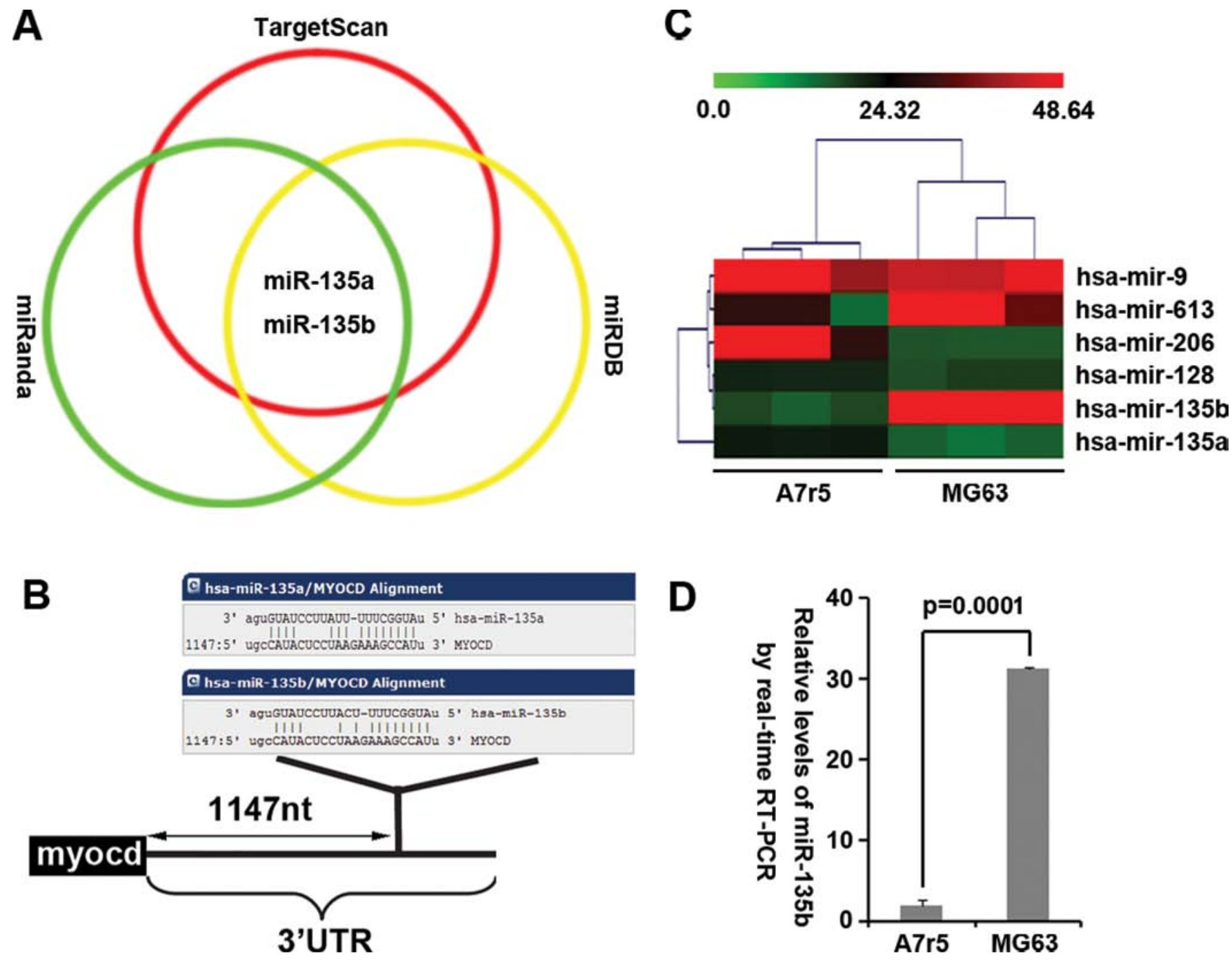

Figure 2. (A) Venn diagram displaying miRNAs computationally predicted to target myocardin by TargetScan (red), miRanda (green) and miRDB (yellow). (B) Predicted miR-135b-binding sites in the myocardin 3'UTR. (C) Partial heat map of miRNA microarray analysis of MG63 cells versus A7r5 cells. n=3. (D) Real-time PCR for miR-135b in A7r5 cells and MG63 cells. U6 was a loading control. $n=3$.

A7r5 cells and MG63 cells. Consistent with the results of microarray, real-time PCR showed that miR-135b expression was significantly upregulated in MG63 cells (Fig. 2D). All the results make us reason that it is probable that downregulation of myocardin was associated with miR-135b overexpression in MG63 cells.

Silencing miR-135b restores the expression of myocardin and miR-135b inhibits myocardin expression by targeting its 3'UTR in osteosarcoma MG63 cells. To examine whether myocardin was indeed regulated by miR-135b, we transfected MG63 cells with anti-miR135b, in which endogenous myocardin is undetectable by RT-PCR and western blotting. Our results showed that anti-miR-135b effectively inhibited miR-135b expression in MG63 cells (Fig. 3A). We next performed RT-PCR and western blotting to detect myocardin expression in MG63 cells transfected with anti-miR-135b. The results showed that myocardin mRNA (Fig. 3B) and myocardin protein (Fig. 3C) were significantly upregulated in MG63 cells transfected with anti-miR-135b. To further demonstrate the direct regulation of myocardin by miR-135b, we constructed luciferase reporters with the targeting sequences of wild-type (myocardin-WT-luc) and mutated myocardin 3'UTRs (myocardin-MUT-luc) (Fig. 3D). Both the wild-type and mutant reporters were introduced into MG63 cells (miR-135b high expression) and A7r5 cells (miR-135b low expression). The luciferase activities of myocardin-WT-luc but not myocardin-MUT-luc were significantly suppressed in MG63 cells but not in A7r5 cells (Fig. 3E).

To detect whether miR-135b targets 3'UTR of myocardin, luciferase assay was performed. Our results showed that miR-135b inhibited myocardin-WT-luc plasmids, but not myocardin-MUT-luc plasmids (Fig. 3F). To further confirm that miR-135b can target the sites of 3'UTR of myocardin as predicated, we transfected MG63 cells with anti-miR-135b. The results demonstrated that silencing miR-135b was able to increase luciferase activity of 3'UTR of myocardin-WTluc in MG63 cells, but not myocardin-MUT-luc (Fig. 3G). All the results indicated that miR-135b directly targeted the sites of 3'UTR of myocardin as predicted by the bioinformatics methods.

In order to further demonstrate that miR-135b inhibits myocardin expression through targeting its predicted sites 
A

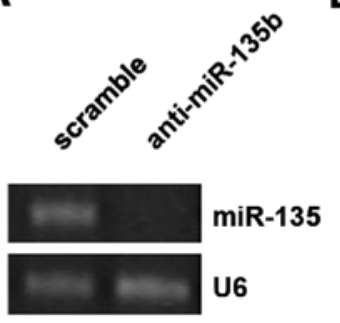

B

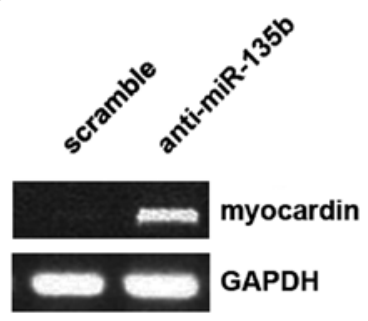

C

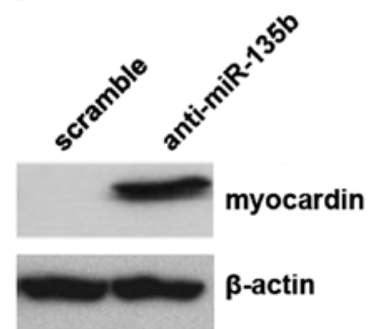

D
3' AGUGUAUCCUUACU - UUUCGGUAU 5' hsa-miR-135b
5' UGCCAUACUCCUAAGAAAGCCAUU 3' myocardin WT 3'UTR
5' UGCCAUACUCCUAAGACGCGGGUU 3' myocardin MUT 3'UTR

E

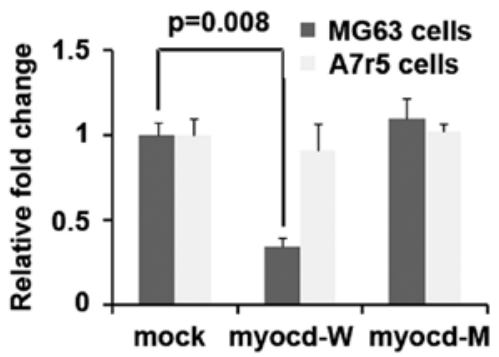

G

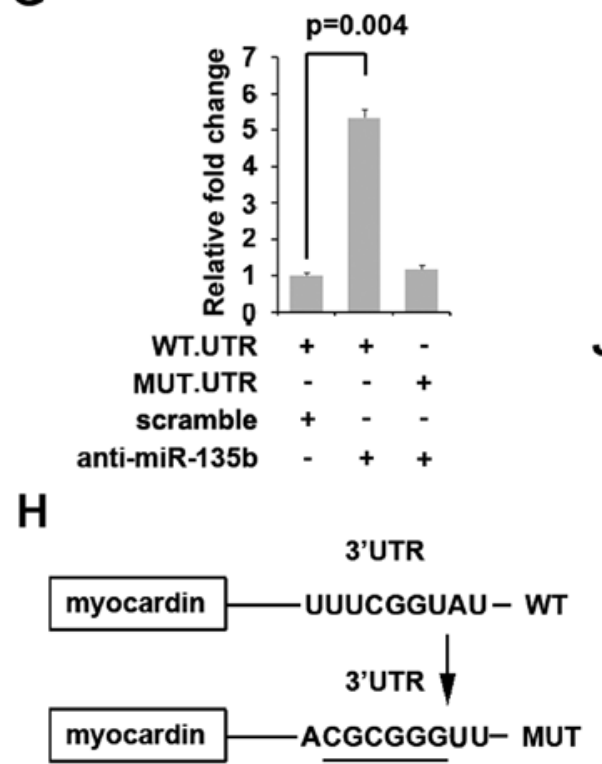

$\mathbf{F}$

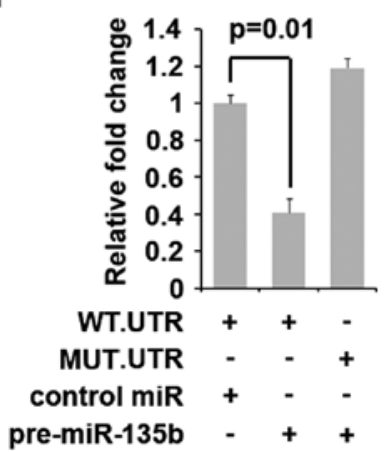

I

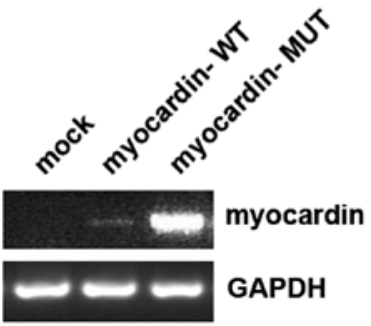

$\mathbf{J}$

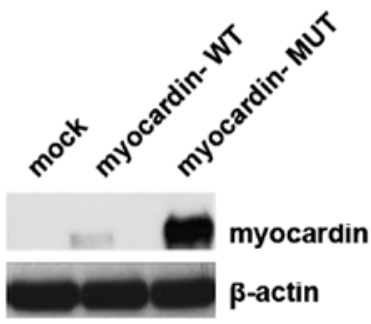

Figure 3. (A) Real-time PCR for miR-135b in MG63 cells. U6 was a loading control. n=3. (B) RT-PCR for myocardin in MG63 cells. GAPDH was a loading control. $n=3$. (C) Western blotting for myocardin in MG63 cells. $\beta$-actin was a loading control. $n=3$. (D) Diagram of myocardin-3'UTR-containing reporter constructs. MUT, contains 6-base-mutation at the miR-135b-target region, abolishing its binding. (E) Reporter assay, WT-UTR but not MUT-UTR reporter activity is reduced only in MG63 cells. n=3. (F) Reporter assay, with cotransfection of 500 ng WT- or MUT-reporter and 50 nM control-miR (scramble), or pre-miR-135b as indicated. $\mathrm{n}=3$. (G) Reporter assay, with cotransfection of $500 \mathrm{ng}$ WT- or MUT-reporter and 50 nM scramble, or anti-miR-135b as indicated. $\mathrm{n}=3$. (H) Diagram of myocardin expression plasmids, containing the predicted target. MUT, contains 6-base-mutation at the miR-135b-target region, abolishing its binding. (I) RT-PCR for myocardin in MG63 cells. GAPDH was a loading control. $\mathrm{n}=3$. (J) Western blotting for myocardin in MG63 cells. $\beta$-actin was a loading control. $n=3$.

of 3'UTR, we purchased myocardin expression plasmids containing predicted sites of 3'UTR (myocardin-WT) and mutated the 6 bases predicted in its 3'UTR (myocardin-MUT)
(Fig. 3H). Both the wild-type and mutant expression plasmids were introduced into MG63 cells (myocardin-negative) and then RT-PCT and western blotting were performed to detect 

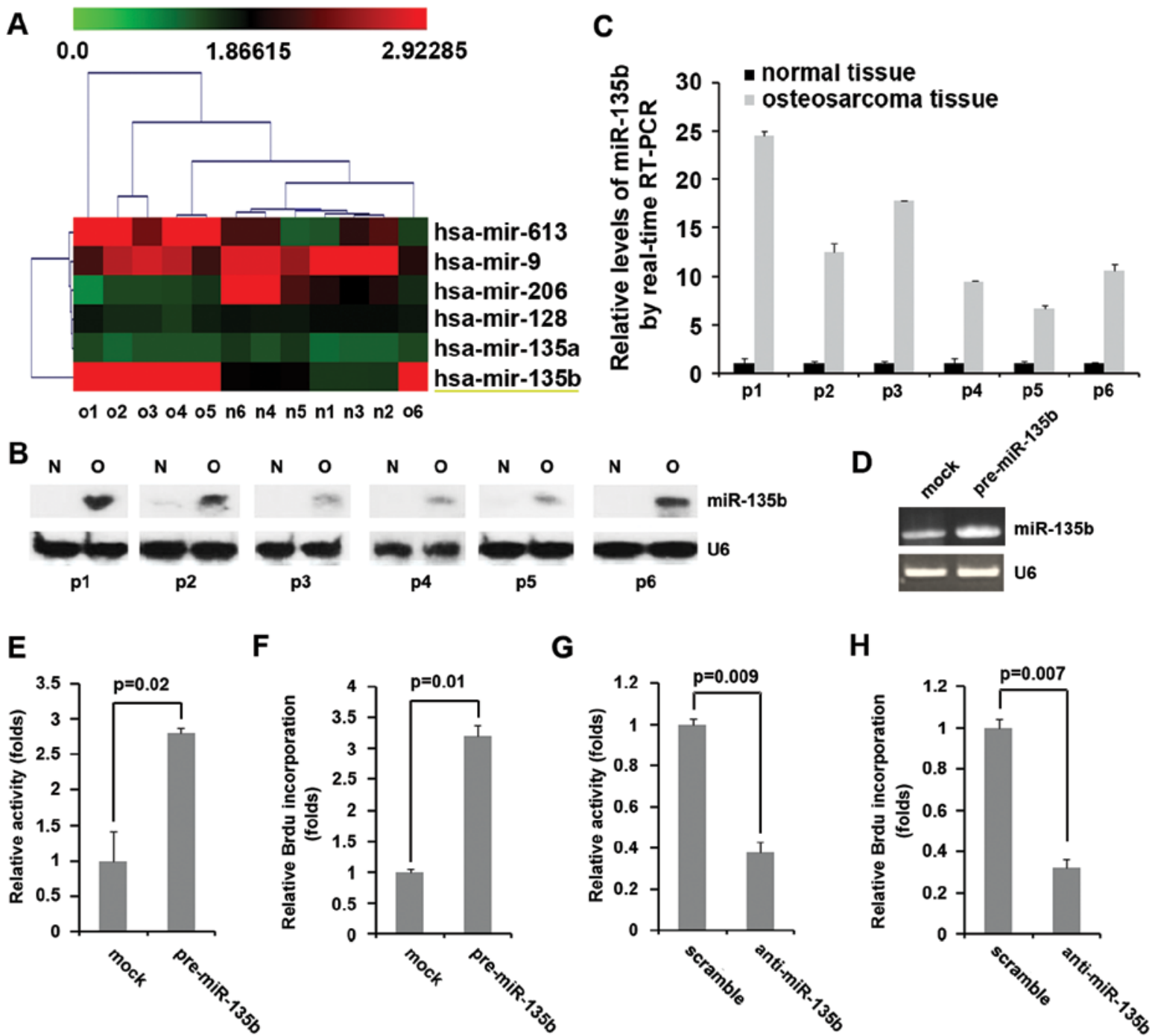

$\mathbf{F}$

G

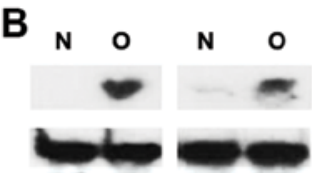

p1

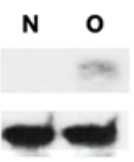

p3
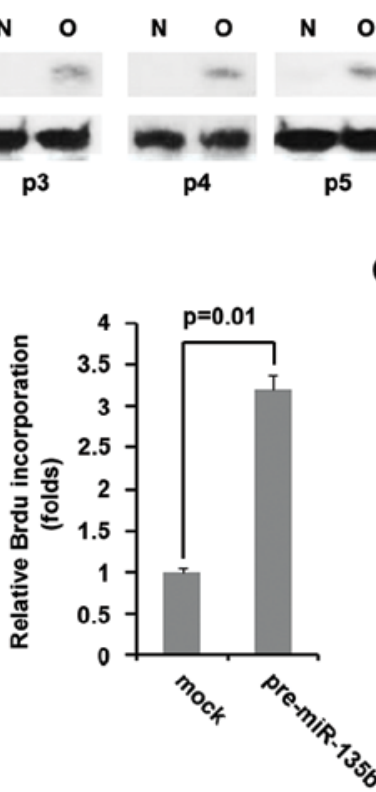

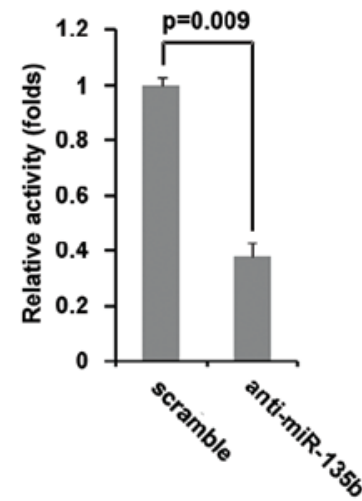

H

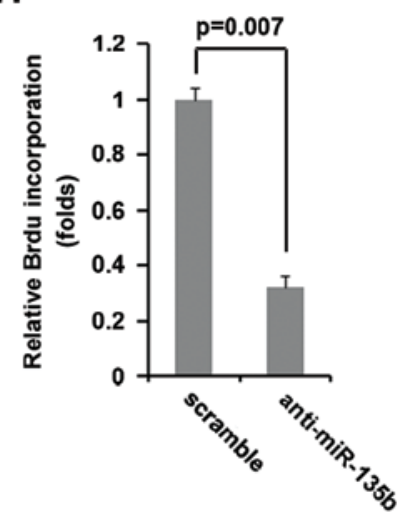

Figure 4. (A) Partial heat map of miRNA microarray analysis of osteosarcoma tissues $(\mathrm{O})$ and normal tissues $(\mathrm{N})$ of 6 patients. $\mathrm{n}=6$. (B) Northern blot analysis using a miR-135b-specific probe showed the level of miR-135b in 6 pairs of osteosarcoma tissues $(\mathrm{O})$ compared with matching controls $(\mathrm{N}$; $\mathrm{P}$, the patient numbers are indicated at the bottom). N=6. (C) Real-time PCR for miR-135b in osteosarcoma tissues (O) and normal tissues (N) of 6 patients. $n=6$. (D) Realtime PCR for miR-135b in MG63 cells. Mock groups were transfected with scramble. n=3. (E) MTT assay for MG63 cells. Mock groups were transfected with scramble. $n=3$. (F) Brdu incorporation for MG63 cells. Mock groups were transfected with scramble. $n=3$. (G) MTT for MG63 cells. $n=3$. (H) Brdu incorporation for MG63 cells. $\mathrm{n}=3$.

A
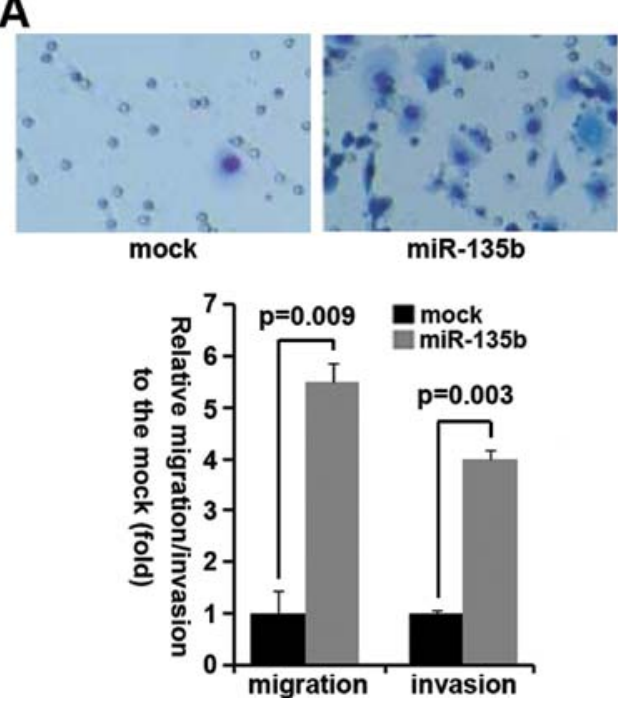

B
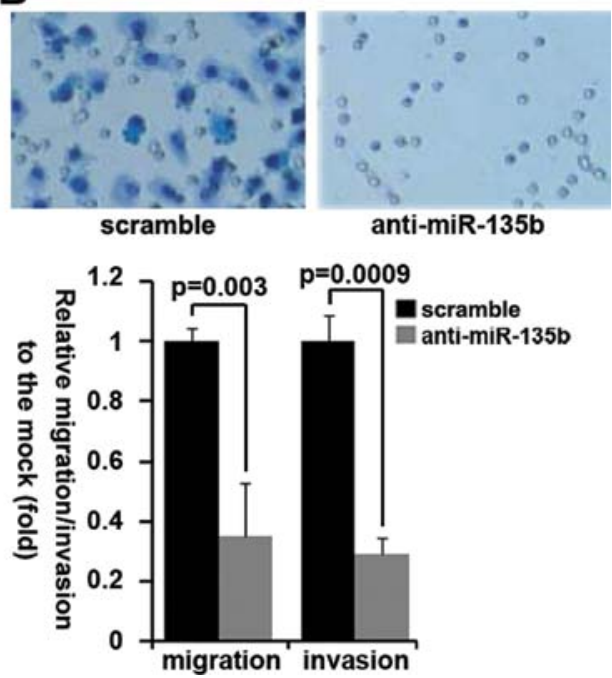

Figure 5. (A) Matrigel invasion assay and Transwell invasion assay for MG63 cells. Mock groups were transfected with scramble. Magnification, $\mathrm{x} 200$. $\mathrm{n}=3$. (B) Matrigel invasion assay and Transwell invasion assay for MG63 cells. Magnification, x200. $n=3$. 
the expression of myocardin. The results showed that only myocardin-MUT significantly increased myocardin mRNA (Fig. 3I) and its protein expression (Fig. 3J) in MG63 cells, indicating that miR-135b indeed inhibited myocardin expression by targeting its $3^{\prime}$ UTR.

miR-135b are highly expressed in osteosarcoma tissues and it promotes MG63 cells proliferation, migration and invasion. Having demonstrated that silencing miR-135b restores the expression of myocardin and miR-135b inhibits myocardin expression by targeting its 3'UTR in MG63 cells. We considered that miR-135b played an important role in MG63 cells. Thus, in an attempt to identify the level of miR-135b expression in osteosarcoma and normal tissues, we performed miRNA profiling in osteosarcoma tissues. RNAs isolated from 6 pairs of osteosarcoma tissue and matched adjacent normal tissue samples were hybridized to a custom miRNA microarray platform. After three hybridizations, quantification, and normalization, we found that miR-135b, was elevated in the primary tumors compared with normal tissues (Fig. 4A).

To further assess the expression of miR-135b in osteosarcoma, northern blot analysis was conducted in 6 pairs of osteosarcoma tissue and matched adjacent normal tissue samples. The expression of miR-135b was consistently higher in osteosarcoma tissues than in normal tissues (Fig. 4B). Consistent with the miRNA microarray data and northern blot analysis, real-time PCR analysis also revealed the expression of miR-135b in the primary tumors compared with normal tissues was elevated (Fig. 4C).

To determine whether miR-135b overexpression would affect cell proliferation, we transfected MG63 cells with pre-miR-135b, and then real-time PCR was performed to detect miR-135b expression in the cells. The results showed that miR-135b expression was effectively increased by pre-miR-135b in MG63 cells (Fig. 4D). MTT assays were performed and the results showed that pre-miR-135b could promote proliferation of MG63 cells (Fig. 4E). Consistent with the results of MTT assay, transfection of MG63 cells with pre-miR-135b increased BrdU incorporation in the cells (Fig. 4F). We had showed that anti-miR-135b could significantly decrease the expression of miR-135b in MG63 cells (Fig. 3A). To further confirm the relationship of miR-135b and proliferation, we performed MTT assay and BrdU incorporation assay and the results showed that silencing miR-135b led to a $>2.5$-fold reduction in the proliferation properties (Fig. 4G) and DNA synthesis of these cells (Fig. 4H).

To determine whether miR-135b would increase the basal levels of cell migration or invasion, we overexpressed miR-135b in MG63 cells. In MG63 cells, ectopic expression of miR-135b resulted in a four- to five-fold increase in cell migration (Fig. 5A) and invasion (Fig. 5A). These results indicated that overexpression of miR-135b is sufficient to promote both migration and invasion in vitro. Consistent with the results of miR-135b overexpression, silencing miR-135b led to $\sim 4$-fold reduction in the migration and invasion of these cells, as gauged by Transwell migration assay (Fig. 5B) and Matrigel invasion assay (Fig. 5B).

Myocardin suppresses proliferation, migration and invasion in MG63 cells. Having demonstrated the miR-135b can promote proliferation, migration and invasion of MG63 cells and that knockdown of miR-135b can restore myocardin expression, we reasoned that myocardin had the opposite effects of miR-135b in MG63 cells. To determine whether myocardin expression in the MG63 cells would also affect cell proliferation, migration and invasion, we performed cell counting assay in MG63 cells transfected with myocardin-MUT. We observed a dosedependent suppression of MG63 cell proliferation (Fig. 6A). Consistent with cell counting assay, MTT assay showed that myocardin suppressed cell proliferation in a dose-dependent manner (Fig. 6B). To further confirm that myocardin can inhibit MG63 cell proliferation, BrdU incorporation assay was performed. The results showed that myocardin inhibited BrdU incorporation in a dose-dependent manner in MG63 cells (Fig. 6C). We next performed gene profiling to search for proliferation-associated genes, and found that CDK1, CDK2, c-myc and Ki67 were significantly downregulated and p27 and p53 were also downregulated in myocardin-MUT transfected groups (Fig. 6D). To further confirm the regulation of CDK1, CDK2, p27, P53, c-myc and Ki67 by myocardin, we performed western blotting to analyze CDK1, CDK2, p27, p53, c-myc, PCNA and Ki67 protein expression in MG63 cells transfected with myocardin-MUT. Our results showed that myocardinMUT significantly inhibited CDK1, CDK2, c-myc and Ki67 protein expression in MG63 cells (Fig. 6E).

We next identified whether restoration of myocardin affected migration and invasion of MG63 cells. Transwell migration assay and Matrigel invasion assay were performed and the results showed that myocardin-MUT significantly inhibited migration and invasion (Fig. 6F).

\section{Discussion}

Deregulated expression of miR-135b in osteosarcoma. miR-135b levels are elevated in a variety of cancers including breast, non-small cell lung cancer (NSCLC), prostate, and colon as well as its upregulation is far more robust in highly invasive lines compared to the less invasive in NSCLC (25-27). Overexpression of miR-135b conferred an increased tumorigenic ability to the relatively benign CL1-0 cells, resulting in $>4$-fold greater tumor burden in xenograft mouse models. In vivo, stable expression of a miR-135b antagonist decreased the number of metastatic tumor nodules in mice injected with highly invasive CL1-5-F4 cells shown to have high levels of miR-135b (15). Clinically, high levels of miR-135b in lung cancer specimens significantly correlated with decreased overall survival (15). Although the expression of miR-135b is upregulated in osteosarcoma and miR-135b functions as an oncogene in other malignant tumors suggesting that it might be a target for malignant tumor therapy, its roles still keep emerging in osteosarcoma. We showed that miR-135b inhibited proliferation, migration and invasion and silencing its expression restored myocardin expression in MG63 cells. Due to the overexpression of miR-135b, myocardin mRNA was degraded in MG63 cells. Next we will study the roles of miR-135b in vivo.

Myocardin as a tumor suppressor in osteosarcoma. Myocardin has been reported to be a key regulator of cardiac and smooth muscle differentiation and its expression is restricted to smooth and cardiac muscle lineages (4). Myocardin drives 
A

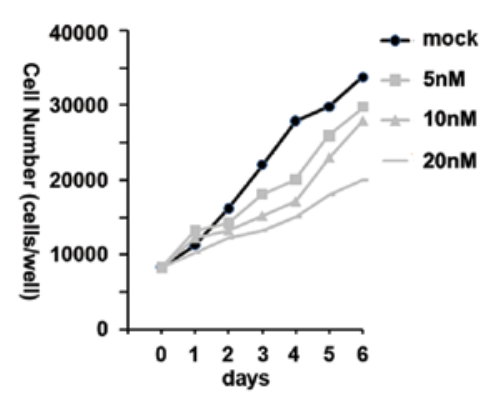

B

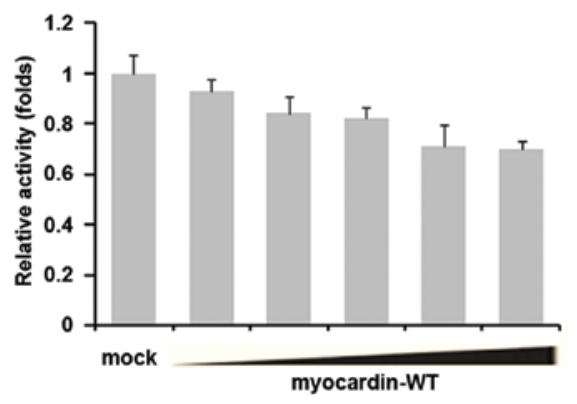

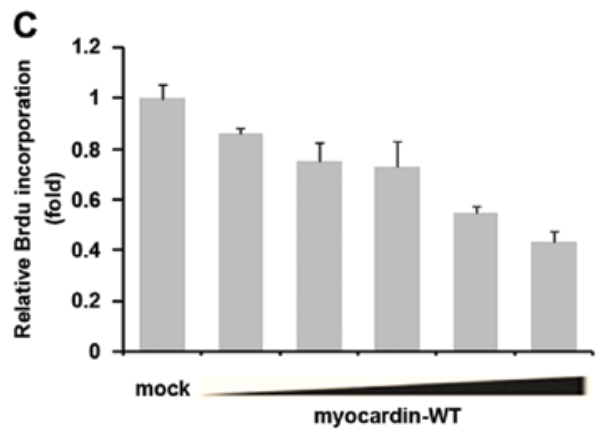

D

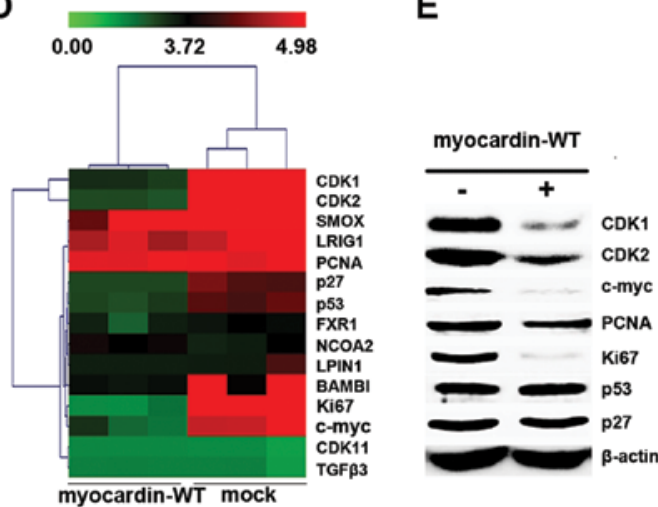

$\mathbf{F}$
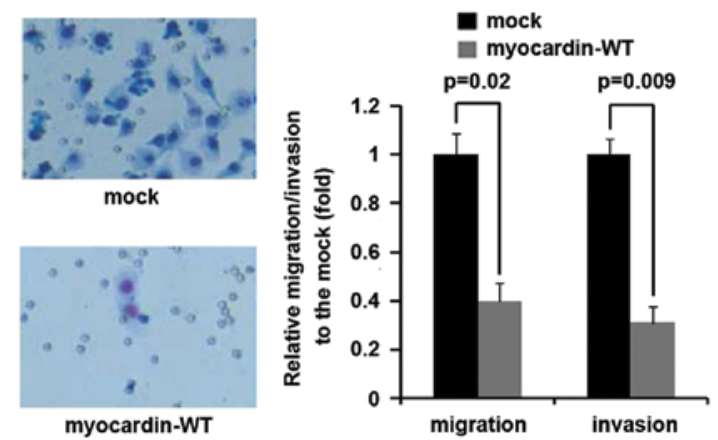

Figure 6. (A) Cell counting assay for MG63 cells transfected with increasing amounts of myocardin. $n=3$. (B) MTT assay for MG63 cells. $n=3$. (C) Brdu incorporation assay for MG63 cells. n=3. (D) Partial heat map of gene microarray analysis. n=3. (E) Western blotting for CDK1, CDK2, c-myc, PCNA, Ki67, p53 and p27 of MG63 cells. $\beta$-actin was a loading control. $n=3$. (F) Matrigel invasion assay and Transwell invasion assay for MG63 cells. Magnification, $x 200$. $n=3$.

transcription through the interaction with the MADS boxcontaining transcription factor, serum response factor (SRF), which binds to a consensus DNA sequence, CC $[\mathrm{A} / \mathrm{T}]_{6} \mathrm{GG}$ (CArG box) $(5,6)$. In addition, myocardin functions as an effective inducer of growth arrest and differentiation of some tumors and is frequently repressed during human malignant transformation (7). Although the importance of myocardin in cardiac and smooth muscle has been firmly established, its role in osteosarcoma is still keep emerging. In this study, we found that myocardin, as a suppressor gene, inhibited proliferation, migration and invasion of MG63 cells. To further confirm the roles of myocardin, its roles in vivo need to be studied.

\section{Reference}

1. Bielack SS, Marina N, Ferrari S, Helman LJ, Smeland S Whelan JS, et al: Osteosarcoma: the same old drugs or more? J Clin Oncol 26: 3102-3103, 2008.

2. Chou AJ, Geller DS and Gorlick R: Therapy for osteosarcoma: where do we go from here? Paediatr Drugs 10: 315-327, 2008.
3. O'Day K and Gorlick R: Novel therapeutic agents for osteosarcoma. Expert Rev Anticancer Ther 9: 511-523, 2009.

4. Wang D, Chang PS, Wang Z, Sutherland L, Richardson JA, et al: Activation of cardiac gene expression by myocardin, a transcriptional cofactor for serum response factor. Cell 105: 851-862, 2001.

5. Yoshida T, Sinha S, Dandré F, Wamhoff BR, Hoofnagle MH, et al: Myocardin is a key regulator of CArG-dependent transcription of multiple smooth muscle marker genes. Circ Res 92: 856-864, 2003.

6. Shore P and Sharrocks AD: The MADS-box family of transcription factors. Eur J Biochem 229: 1-13, 1995.

7. Pipes GC, Creemers EE and Olson EN: The myocardin family of transcriptional coactivators: versatile regulators of cell growth, migration, and myogenesis. Genes Dev 20: 1545-1556, 2006.

8. Lulla RR, Costa FF, Bischof JM, Chou PM, de F Bonaldo M, Vanin EF and Soares MB: Identification of Differentially expressed microRNAs in osteosarcoma. Sarcoma 2011: 732690, 2011.

9. Jones KB, Salah Z, Del Mare S, Galasso M, Gaudio E, Nuovo GJ, Lovat F, LeBlanc K, Palatini J, Randall RL, Volinia S, Stein GS, Croce CM, Lian JB and Aqeilan RI: miRNA signatures associate with pathogenesis and progression of osteosarcoma. Cancer Res 72: 1865-1877, 2012. 
10. Zhang H, Cai X, Wang Y, Tang H, Tong D and Ji F: microRNA-143, down-regulated in osteosarcoma, promotes apoptosis and suppresses tumorigenicity by targeting Bcl-2. Oncol Rep 24: $1363-1369,2010$

11. Duan Z, Choy E, Harmon D, Liu X, Susa M, Mankin H and Hornicek F: MicroRNA-199a-3p is downregulated in human osteosarcoma and regulates cell proliferation and migration. Mol Cancer Ther 10: 1337-1345, 2011.

12. Osaki M, Takeshita F, Sugimoto Y, Kosaka N, Yamamoto Y, Yoshioka Y, Kobayashi E, Yamada T, Kawai A, Inoue T, Ito H, Oshimura M and Ochiya T: MicroRNA-143 regulates human osteosarcoma metastasis by regulating matrix metalloprotease-13 expression. Mol Ther 19: 1123-1130, 2011.

13. Ziyan W, Shuhua Y, Xiufang W and Xiaoyun L: MicroRNA-21 is involved in osteosarcoma cell invasion and migration. Med Oncol 28: 1469-1474, 2011.

14. Arigoni M, Barutello G, Riccardo F, Ercole E, Cantarella D, Orso F, Conti L, Lanzardo S, Taverna D, Merighi I, Calogero RA, Cavallo $\mathrm{F}$ and Quaglino E: miR-135b coordinates progression of ErbB2-driven mammary carcinomas through suppression of MID1 and MTCH2. Am J Pathol 182: 2058-2070, 2013.

15. Lin CW, Chang YL, Chang YC, Lin JC, Chen CC, Pan SH, Wu CT, Chen HY, Yang SC, Hong TM and Yang PC: MicroRNA-135b promotes lung cancer metastasis by regulating multiple targets in the Hippo pathway and LZTS1. Nat Commun 4: 1877, 2013.

16. Zhang L, Sun ZJ, Bian Y and Kulkarni AB: MicroRNA-135b acts as a tumor promoter by targeting the hypoxia-inducible factor pathway in genetically defined mouse model of head and neck squamous cell carcinoma. Cancer Lett 331: 230-238, 2013.

17. Matsuyama H, Suzuki HI, Nishimori H, Noguchi M, Yao T, Komatsu N, Mano H, Sugimoto K and Miyazono K: miR-135b mediates NPM-ALK-driven oncogenicity and renders IL-17producing immunophenotype to anaplastic large cell lymphoma. Blood 118: 6881-6892, 2011.

18. Khatri R and Subramanian S: MicroRNA-135b and its circuitry networks as potential therapeutic targets in colon cancer. Front Oncol 3: 268, 2013.
19. Holycross BJ, Blank RS, Thompson MM, Peach MJ and Owens GK: Platelet-derived growth factor-BB-induced suppression of smooth muscle cell differentiation. Circ Res 71: $1525-1532,1992$.

20. Luo XG, Zou JN, Wang SZ, Zhang TC and Xi T: Novobiocin decreases SMYD3 expression and inhibits the migration of MDA-MB-231 human breast cancer cells. IUBMB Life 62: 194-199, 2010.

21. Liao XH, Wang N, Liu QX, Qin T, Cao B, et al: Myocardin-related transcription factor-A induces cardiomyocyte hypertrophy. IUBMB Life 63: 54-61, 2011.

22. Frasor J, Danes JM, Komm B, Chang KC, Lyttle CR and Katzenellenbogen BS: Profiling of estrogen up- and downregulated gene expression in human breast cancer cells: insights into gene networks and pathways underlying estrogenic control of proliferation and cell phenotype. Endocrinology 144: 4562-4574, 2003.

23. Yu J, Ryan DG, Getsios S, Oliveira-Fernandes M, Fatima A and Lavker RM: MicroRNA-184 antagonizes microRNA-205 to maintain SHIP2 levels in epithelia. Proc Natl Acad Sci USA 105: 19300-19305, 2008.

24. Lu J, Getz G, Miska EA, Alvarez-Saavedra E, Lamb J, Peck D, Sweet-Cordero A, Ebert BL, Mak RH, Ferrando AA, Downing JR, Jacks T, Horvitz HR and Golub TR: MicroRNA expression profiles classify human cancers. Nature 435: 834-838, 2005.

25. Bandres E, Cubedo E, Agirre X, Malumbres R, Zarate R, Ramirez N, et al: Identification by real-time PCR of 13 mature microRNAs differentially expressed in colorectal cancer and non-tumoral tissues. Mol Cancer 5: 29, 2006.

26. Lowery AJ, Miller N, Devaney A, Mcneill RE, Davoren PA, Lemetre C, et al: MicroRNA signatures predict oestrogen receptor, progesterone receptor and HER2/neu receptor status in breast cancer. Breast Cancer Res 11: R27, 2009.

27. Tong AW, Fulgham P, Jay C, Chen P, Khalil I, Liu S, et al: MicroRNA profile analysis of human prostate cancers. Cancer Gene Ther 16: 206-216, 2013. 